\title{
Modeling Method and Application of College Comprehensive Teaching Mode Based on Artificial Intelligence
}

\author{
Yin Wenjing \\ Zhengzhou Preschool Education College, Zhengzhou, Henan, China
}

\begin{abstract}
Under the influence of artificial intelligence, education is undergoing a profound innovation. On the basis of expounding the important position of artificial intelligence in the field of computer, this paper studies the modeling method of college comprehensive teaching mode based on artificial intelligence. This paper analyzes the feasibility of applying the task driven method to the teaching of artificial intelligence course based on the teaching objectives of artificial intelligence course and the characteristics and steps of task driven teaching method. This paper proposes a task driven teaching model of artificial intelligence. Then, this paper explores the specific shift process of instructional design paradigm under the background of artificial intelligence. In the pre intelligence stage, this study uses the paradigm shift analysis framework to verify whether Cai instructional design, multimedia instructional design and information instructional design have migration. For the teaching design in the intelligent stage, this paper first expounds the inevitability of the paradigm shift of teaching design under the background of artificial intelligence from the aspects of the opportunities brought by artificial intelligence to education and teaching, the problems of the original information-based teaching design and the many challenges it faces. At last, the typical application cases under the guidance of intelligent instructional design paradigm are described in detail.
\end{abstract}

Keywords: Artificial Intelligence, Teaching Mode, Modeling Method, Migration Analysis.

\section{Introduction}

Artificial intelligence provides a lot of opportunities for the development of many fields, and its important role has attracted great attention at home and abroad. Many countries have formulated policies and plans to upgrade artificial intelligence into a national strategy. The United States has promulgated "preparing for the future of artificial intelligence" and "national artificial intelligence R\&D strategic plan" [1-2]. The European Commission has drawn up SPARC robot innovation plan, the British and German governments have formulated "modern industry strategy" and "industry 4.0" plans respectively, and the Korean government has established AI star lab in 2015. Artificial intelligence has become one of the five key areas of national research. The Japanese government has planned the route of AI industrialization and deployed the super intelligent society [3]. Our government has also introduced plans for the development of the new generation of AI industry, such as the three year action plan to promote the development of the new AI industry (2018 one 2020), the Internet plus artificial intelligence three year action plan, the new generation AI development plan, and the promotion of big data development program.

The promulgation of a series of policies at home and abroad marks that human beings are going to the era of wisdom [4-5]. Our current education is an extension of the industrial age, is the education of known knowledge, is to cultivate knowledge people, skills for the purpose of education. This can not meet the requirements of social development, we need to build a new education system [6]. In addition, with the opportunities brought by artificial intelligence, researchers in the field of education also need to promote the reform and innovation of education and teaching, and realize the integrated development of technology and education. 


\section{The influence of artificial intelligence on the elements of instructional design}

\subsection{Related concepts of artificial intelligence}

Artificial intelligence is a comprehensive subject that studies the use of computers to imitate and expand the function of human brain. The research on artificial intelligence originated from Turing's assumption in 1950: can machines really think? The widely recognized origin of artificial intelligence was the Dartmouth conference in 1956. Recently, artificial intelligence rose again in 2006 due to the success of deep learning algorithm. According to the strength of artificial intelligence, it can be divided into weak artificial intelligence, strong artificial intelligence and super artificial intelligence. Weak artificial intelligence refers to the artificial intelligence that is only good at a certain aspect, mainly the dominant intelligence of human beings [7-10]. The current research of artificial intelligence focuses on this type. Strong artificial intelligence is a kind of artificial intelligence at human level, which can be compared with human beings and possess and manifest explicit intelligence.

Artificial intelligence has different application forms in the field of education, and different researchers have different division of artificial intelligence system in the field of education. One view is that the artificial intelligence system in the field of education mainly includes intelligent teaching system, intelligent agent, intelligent question answering system and intelligent decision support system. Some scholars divide AI into intelligent tutor system, automatic evaluation system, educational game and educational robot. Combined with the above application form classification, the author based on Zhang Kunying's integration mode of artificial intelligence technology and education. As well as Zhong Zhixian's support form for technology in the framework of instructional design, the application form of artificial intelligence in education is divided into two forms: subjectivity and assistance, as shown in Figure 1.

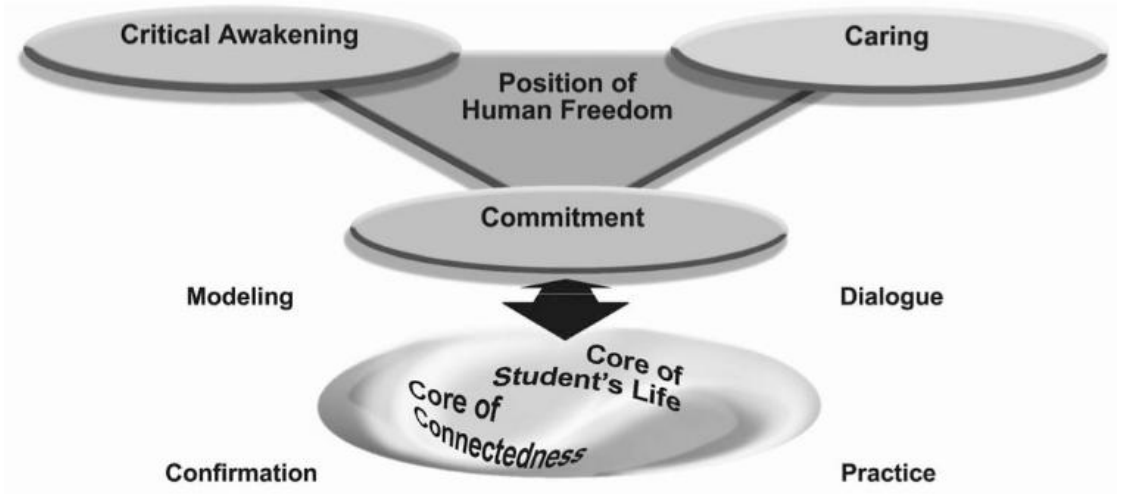

Fig 1: Application form of artificial intelligence in teaching system

At present, although the application of artificial intelligence technology in education is at the initial stage, artificial intelligence technology is the most revolutionary technology at this stage. Technology is not only a tool of education and teaching, but also a revolutionary factor to subvert education and teaching. However, not all technological revolutions will completely change education. Only some revolutionary technologies will have such an impact. With the maturity of artificial intelligence technology, the learning ability of machine is stronger and stronger, and its application in the field of education will be more and more in-depth.

2.2 The general meaning of instructional design

The professional backgrounds of the designers of teaching system are different, including primary and secondary school and vocational education teachers, media technicians, subject experts, managers and teaching system design 
professionals. At present, there are four different understandings of the connotation of teaching design. One understanding is that instructional design is a process of systematic planning or preparation for teaching, represented by Gagne, Kemp, Reagan, umena and he Kekang. The second understanding is represented by Merrill, who believes that instructional design is the secondary development technology of past learning experience and objective environment. The third understanding regards instructional design as a design science in the field of education, and the most representative one is Patton's what is instructional design. Some scholars understand instructional design as a performance optimization technology. Generally speaking, the most basic and necessary aspect of teaching system design lies in the continuity of teaching design, that is, teaching design is a continuous "action" or "process", not just a certain result or effect.

Combined with the disciplines involved in this study, this study adopts the definition of instructional design by he Kekang, which is highly recognized in China. Instructional design is the use of systematic methods to transform the principles of learning theory and teaching theory into specific plans for teaching objectives, teaching contents, teaching methods and teaching strategies, teaching evaluation and other links, and to create a systematic "process" or "program" of teaching and learning. Teaching design is different from teaching theory. Discipline theory, which directly guides teaching theory, plays an indirect role in teaching design, while technical rationality plays a direct role. In addition, it is aimed at the design and technology activities in the teaching process, rather than mainly studying the nature and laws of teaching.

Teaching design integrates all the elements of teaching system, and takes the teaching system as a whole to plan, implement and evaluate, so as to make it a system with the best function. However, the elements of the teaching system are affected by the background of the times, the theoretical basis and the technical tools. The elements of the teaching system change dynamically, including three elements, four elements, five elements, six elements, seven elements, and the elements of composition and process. The foresight of instructional design on teaching activities can effectively determine the teaching objectives, teaching content, teaching methods, teaching process and so on. It must face up to the new changes and demands of teaching elements under the background of artificial intelligence, and give full play to the advantages of intelligent technology in improving the quality of teaching and promoting the development of talents.

\section{The transfer of instructional design paradigm}

\subsection{Paradigm transfer analysis framework}

Kuhn believes that the scientific revolution is not a process of gradual accumulation, but a systematic process of "paradigm transfer" of the whole scientific research. In essence, it is a new paradigm replacing the old paradigm. After analyzing the meaning of paradigm and scientific community, he proposed the growth model of scientific knowledge to integrate the development of Science: pre discipline (no paradigm) - conventional Science (paradigm construction) - Scientific Revolution (paradigm shaking) - new conventional Science (new paradigm formation). Therefore, the paradigm is not static, but constantly improved and expanded through application. In Kuhn's words, paradigm is an object to be further clarified and improved in the new and urgent environment. In this sense, after the initial formation of a certain paradigm, the work of follow-up researchers usually need to add new content to the paradigm to make it more perfect and substantial. But as time goes on, some new paradigms will appear in a competitive way. Through the new practical choice, the new paradigm may replace the old paradigm and become the recognized paradigm of the discipline. We call this phenomenon paradigm transfer.

In order to make the outside world have a clearer understanding of the concept of paradigm and provide relevant researchers with a reference system to judge the emergence of new disciplines or the disappearance of old paradigm, Kuhn designed a subject appraisal table including four indicators, namely, the framework of paradigm transfer to determine whether the paradigm has been transferred. The four indicators are: symbol generalization, belief, value and example promised by members of scientific community. Through the analysis of paradigm and 
paradigm transfer analysis framework, this study intends to use four elements of Kuhn about paradigm, including symbol generalization, belief, value and paradigm promised by community members as the analysis tools for judging paradigm transfer, as shown in Figure 2, which provides the basis for judging whether paradigm transfer is produced in Teaching design.

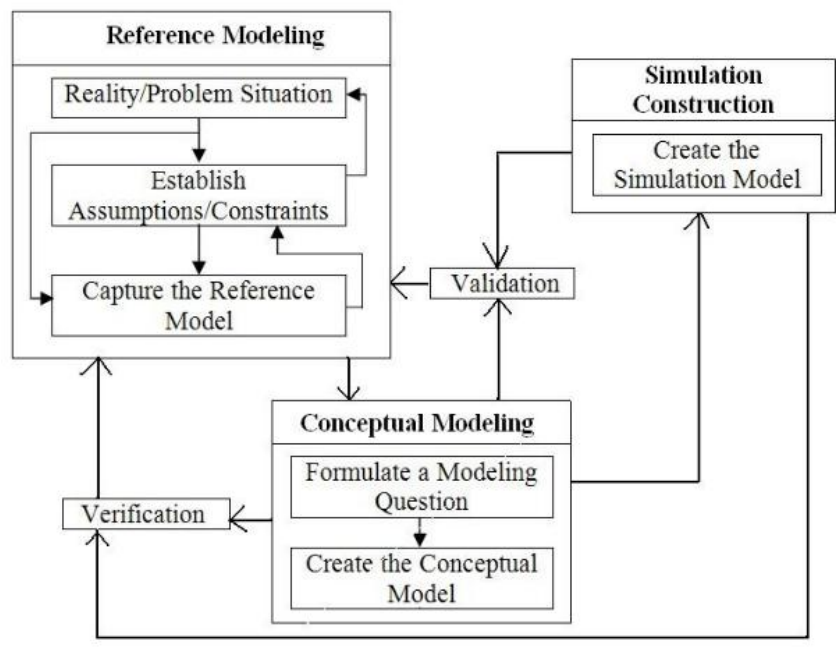

Fig 2: Paradigm transfer analysis framework

\subsection{Instructional design paradigm and its transfer connotation}

Since instructional design paradigm exists, it has been discussed. What is the meaning of instructional design paradigm? Through the analysis of academic papers related to instructional design paradigm, it is found that many scholars have put forward different instructional design paradigms according to their solid professional background, but unfortunately few scholars have given a clear definition of what is instructional design paradigm.

Throughout the development of instructional design, teaching theory, system theory, communication theory and learning theory have a profound impact on it. However, the theoretical system of the first three has developed steadily in the past 30 years, and has no obvious effect on the development of instructional design. Only learning theory, through the development stages of behaviorism, cognitivism and constructivism, promotes the continuous progress of instructional design.

Based on the analysis of the existing instructional design paradigm, combined with the concepts of instructional design and paradigm, this paper believes that instructional design paradigm is the basic consistent way of thinking and means of behavior in solving instructional design problems, which is upheld by the instructional design community, and provides the theoretical model and method framework of Instructional design for community members. According to the application of Kuhn's paradigm transfer theory in the field of natural science, paradigm transfer means that the new paradigm replaces the old paradigm and constantly competes in the process of scientific revolution. However, the transfer of instructional design paradigm does not completely follow this process. When a certain instructional design paradigm occupies a dominant position, it enters the "conventional science" period. In this period, the work of instructional design researchers is to expand and enrich this paradigm.

In addition, instructional design is not the discovery of new laws or laws in nature, but the application of existing laws theory and technology to promote teaching innovation. It will form different theories due to researchers' diverse research perspectives, purposes and methods. In the process of its development, it will be affected by different theories, ideas and technology development, and eventually produce many different paradigms. These paradigms share common research methods, ideas and guiding examples. Each paradigm definitely clarifies one 
part of instructional design activities, and intentionally or unintentionally avoids the rest. However, each paradigm occupies a specific dominant position in a certain range of a certain historical period, and has its own stable way of solving problems.

\section{Transfer of instructional design paradigm under the background of artificial intelligence}

\subsection{The inevitability of paradigm transfer of instructional design under the background of artificial intelligence}

(1) The fourth paradigm closely related to big data the transformation of data intensive science to the paradigm of instructional design

The fourth paradigm data intensive science uses data science to collect, store, analyze and manage data. Under the influence of the fourth paradigm, data intensive science, artificial intelligence, big data and other technologies, paradigm changes have taken place from social science research to education and teaching, and then to educational technology. Kuhn pointed out that "the transformation of scientific paradigm is essentially the revolution of the world outlook of scientific community", so it is reasonable to believe that the transformation of social science research paradigm, teaching paradigm and educational technology paradigm also means the revolution of the world outlook shared by the community of corresponding research levels. Instructional design is a subset of the research field of educational technology. The outer part of instructional design has undergone a revolutionary change at the paradigm level, while the inner part of instructional design must have the necessity of paradigm transfer.

(2) The influence of artificial intelligence technology on learning theory involved in Instructional Design

Zhu zhiting pointed out that "the theoretical basis of cultivating smart talents in the smart learning ecosystem has changed from the connectivism theory of the digital learning ecosystem to the smart education theory". Based on the analysis of ID1 and ID2, Tan Wei thought that intelligent instructional design might become a new direction of instructional design. Although the definition and structure of folding instructional design theory has not yet appeared, the sprout of intelligent instructional design in the research of instructional design theory has appeared. From the above analysis, it is found that artificial intelligence technology will indeed have a significant impact on the development of a theoretical foundation of instructional design. In addition, the symbolic generalization in the elements of the paradigm is abstracted from a set of theoretical categories shared by the members of the community. Logically speaking, the change of theoretical basis will cause the change of symbolic generalization, which makes the transfer of new instructional design paradigm inevitable.

\subsection{Transfer of instructional design paradigm under the background of artificial intelligence}

Under the premise of analyzing the inevitable transfer of teaching design paradigm in the background of artificial intelligence, this section will summarize its key features from the four elements of paradigm composition to determine whether it will be transferred.

1. The commitment of community members

Artificial intelligence technology has a far-reaching impact on the various elements of the teaching system, which integrates and surpasses the previous technology to assume the role of teaching media and teaching tools in the design of teaching system. Therefore, the belief of new instructional design paradigm combines the media view of multimedia instructional design and the tool view of information instructional design, which is called ecological view in this study. At first, ecological view refers to the general understanding of ecological problems, which guides human to understand and improve nature. Ecosystem is a system formed by the interaction of organisms and abiotic components in the environment in a certain space. The basic concept of ecological concept has been used in various application fields, which has universality. 
2. Values agreed by the members of the community

The core value shared by the members of the community of multimedia instructional design paradigm is to take instructional design as a kind of transmission technology, and transfer objective knowledge and skills to learners to maximize the effectiveness of teaching. The value of information-based instructional design lies in taking learners as the center, encouraging learners to participate actively and achieving the optimization of the teaching process. Although the latter plays a positive role in promoting educational informatization and innovation, neither of them has a revolutionary impact on school education and teaching. In the context of artificial intelligence, the biggest advantage of instructional design is to make full use of the advantages of big data, cloud computing, artificial intelligence and other intelligent technologies to build an ecological teaching system integrating technology, so as to improve the personalization and accuracy of education and teaching.

3. A set of "symbolic generalization" shared by community members

By analyzing the inevitability of paradigm transfer of instructional design under the background of artificial intelligence, we know that artificial intelligence technology will have an impact on the theoretical basis of instructional design. For example, Zhu zhiting put forward the theory of intelligent education. Tan Wei and Wu Pengze believe that new technologies such as artificial intelligence and big data will promote the emergence of new learning theories, but no scholars have systematically analyzed the specific connotation of the theoretical basis. Therefore, this study attempts to abstract the common symbols of community members under the background of artificial intelligence by combining the study of the meaning of paradigm and the two major learning theories in the Internet age, connectionism and Neo constructivism.

4. Examples shared by community members that can be imitated

For example, the robot center of Chuiqing Institute of green intelligent technology of Chinese Academy of Sciences has developed a humanoid service robot into a teaching robot, initially constructed a robot aided teaching mode based on constructivism, and proved the effectiveness of the teaching design mode."Knetp2010" puts forward the connected teaching model based on technology, which aims to rebuild an effective teaching model. It hopes that with the help of technology, anyone can connect learners, educators, learning tools, learning resources and learning environment anytime and anywhere. To obtain the most effective, simple and powerful learning support and service, and artificial intelligence technology is the technical basis for this purpose.

\subsection{Typical cases of intelligent teaching design}

Case study: active learning and Knewton adaptive learning system of Arizona State University

Since 2011, the teaching model based on bloom classification has been adopted by the teachers of Arizona State University, which overturns the traditional teaching method relying on teaching. The intelligent teaching application of the combination of adaptive technology and active learning is introduced, as shown in Figure 3. At the bottom of the pyramid, the machine is the main body, mainly using artificial intelligence technology and adaptive technology to help students complete some rote learning. At the top of the pyramid, teachers are the main body, mainly carrying out the learning and communication of higher-level ability with students.

After adaptive learning technology is embedded in teaching activities, learners can carry out efficient learning according to their own rhythm, and choose some learning content they are interested in. At the same time, in the form of active learning to carry out classroom teaching, learners and teachers or other students face-to-face communication, better solve the problem. Teachers mainly guide students to think, and do not provide fixed answers. Active learning has a high demand for teachers. When teachers use the traditional form of lectures, teachers control the whole classroom. There is only one purpose of teachers, that is, the transmission of information.In active learning, the classroom should still be under the control of teachers, but the perspective of classroom conversation communication can develop in many directions, which may lead to chaos of control. 
In this teaching process, technology has an impact on the subject and object of instructional design elements with its form of productivity. The subject and object of teaching has changed from teachers and students to teachers, students and artificial intelligence. Through the interactive form of human-computer integration, teachers have abundant energy to implement effective teaching methods, and learners can obtain personalized learning services. Through the construction of man-machine integration of the ecological environment, improve the personalized and accurate education and teaching, and gradually realize the deep integration of technology and education.

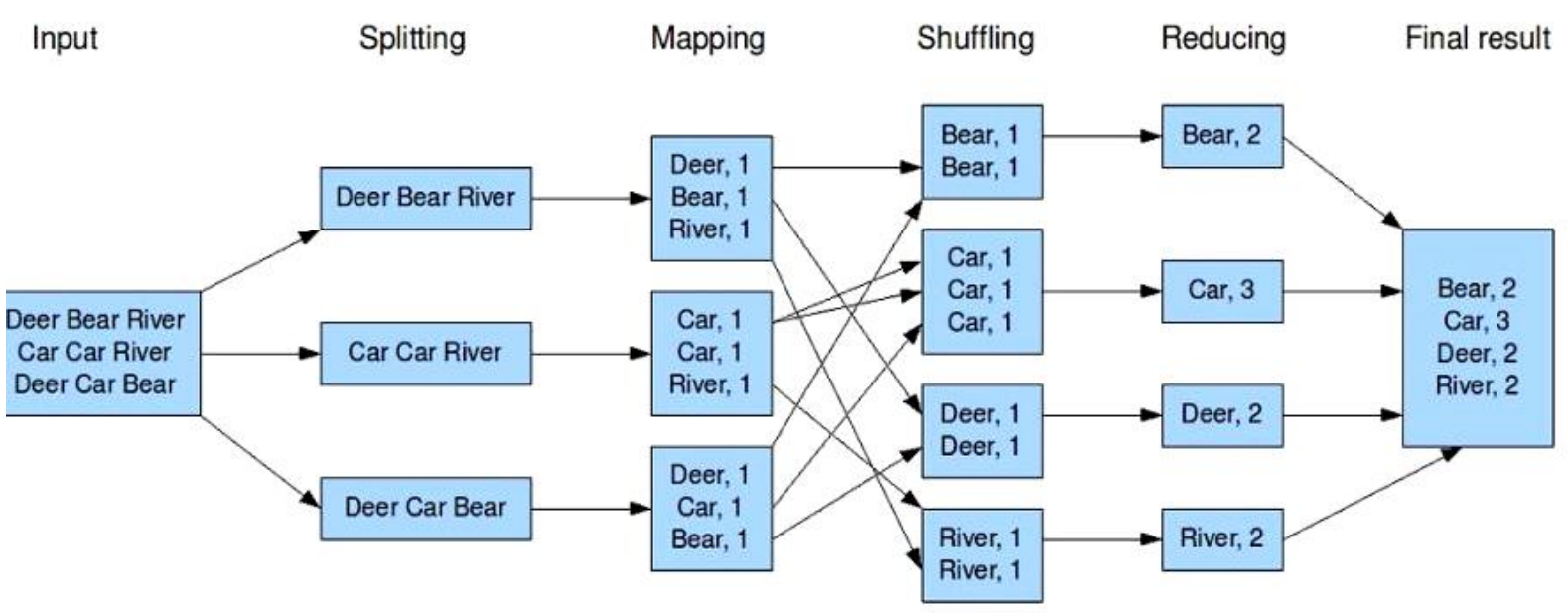

Fig 3: Application hierarchy of intelligent education in Arizona State University

By 2018, the experiment of AI technology in the school has been successful. In this teaching system, teachers and adaptive learning systems play the role of teaching subjects respectively. Over the past seven years, more than 65000 students at Arizona State University have used adaptive learning systems and 12 courses have also used adaptive systems to some extent. In the introduction course of biology in this university, the former teaching was carried out in the form of teaching, with the dropout rate hovering around $10 \%$, and about $77 \%$ of students reached $\mathrm{C}$ or above. Now, through adaptive technology and teachers' collaborative role in teaching activities, students' enthusiasm for learning has generally improved, and the drop out rate in spring 2016 has dropped to 5\%, and 91\% of students have a score of $\mathrm{C}$ or higher.

\section{Conclusion}

This study explores the influence of artificial intelligence on the elements of instructional design one by one, and uses the paradigm transfer analysis framework to prove the paradigm transfer of instructional design under the background of artificial intelligence, and puts forward a paradigm transfer model from multimedia instructional design to Information Instructional Design and then to intelligent instructional design. On this basis, it also analyzes the typical cases of intelligent instructional design paradigm for practice guidance. In addition, this paper also has the shortcomings of the research, the key points of the implementation of intelligent instructional design paradigm and the future expectations of artificial intelligence education.

\section{References}

[1] Yoshikawa, N , et al. "Design and component test of a tiny processor based on the SFQ technology." IEEE Transactions on Applied Superconductivity, vol.13, no.2, pp. 441-445, 2003.

[2] Tong, Rongbai, et al. "Recent research progress in the synthesis and properties of burning rate catalysts based on ferrocene-containing polymers and derivatives." Journal of Organometallic 
Chemistry, vol.755, no.2, pp. 16-32, 2014.

[3] Shimada, Izumi, and J. F. Merkel . "Copper-Alloy Metallurgy in Ancient Peru." entific American, vol.265, no.1, pp. 80-861991.

[4] Luger A.. "Advances in Blue OLEDs Increase Efficiency and Lifetime." Critical Care Medicine, vol.14, no.5, pp. 458-461, 1986.

[5] Cavallaro, Rauno, L. Demasi, A. Passariello. "Nonlinear Analysis of PrandtlPlane Joined Wings: Effects of Anisotropy." AIAA Journal, vol.52, no.5, pp. 964-980, 2014.

[6] Segal, M. M. , et al. "Evidence-based decision support for neurological diagnosis reduces errors and unnecessary workup. " Journal of Child Neurology, vol.29, no.4, pp. 487-92, 2014.

[7] Lindqvist, Olav, and C. Tishelman . "Room for Death - International museum-visitors' preferences regarding the end of their life." Social Science \& Medicine, vol.139, no.2, pp. 1-8, 2015.

[8] Chen Lei, Li Miao, Zhang Jian. Research on Ordering Method of Chinese Mongolian Statistical Machine Translation with Limited Corpus. Chinese Journal of Information Technology, 2013 (05): 202-208

[9] Lu Xuemei, Cheng Lidong, Zhang Hong. the Idea of Improving the International Display of Chinese Sci-tech Journals Based on Online Translation System. Journal of Chinese Science and Technology, 2018, 30 (2):33-38

[10] Wang Haifeng, Li Sheng, Zhao Tiejun. Processing Strategies of Chinese Clutch Words in Chinese English Machine Translation. Acta Informatics Sinica, 1999, 18 (4): 303-307 